This document is confidential and is proprietary to the American Chemical Society and its authors. Do not copy or disclose without written permission. If you have received this item in error, notify the sender and delete all copies.

\title{
A nanoscale investigation on the influence of anodization parameters during plasma electrolytic oxidation of titanium by high-resolution electron energy loss spectroscopy
}

\begin{tabular}{|r|l|}
\hline Journal: & ACS Applied Materials \& Interfaces \\
\hline Manuscript ID & Draft \\
\hline Manuscript Type: & Article \\
\hline Date Submitted by the & n/a \\
\hline Complete List of Authors: & $\begin{array}{l}\text { Casanova, Luca; Politecnico di Milano, Dept. Chemistry, Materials and } \\
\text { Chemical Engineering "G. Natta" } \\
\text { Arosio, Mattia; Politecnico di Milano } \\
\text { Hashemi, Mohammad Taghi; McMaster University } \\
\text { Pedeferri, Mariapia; Politecnico di Milano, CMIC } \\
\text { Botton, Gianluigi A.; McMaster University, Materials Science and } \\
\text { Engineering } \\
\text { Ormellese, Marco; Materiali e Ingegneria Chimica "G. Natta", } \\
\text { Dipartimento di Chimica }\end{array}$ \\
\hline $\begin{array}{l}\text { Note: The following files were submitted by the author for peer review, but cannot be converted to PDF. } \\
\text { You must view these files (e.g. movies) online. }\end{array}$ \\
\hline manuscript.docx
\end{tabular}

\section{SCHOLARONE" Manuscripts}

